\title{
A Closed Form Solution to a Special Normal Form of Riccati Equation
}

\author{
Haiduke Sarafian \\ University College, The Pennsylvania State University, York, USA \\ E-mail: has2@psu.edu \\ Received May 18, 2011; revised June 28, 2011; accepted July 10, 2011
}

\begin{abstract} $n$.

Keywords: Riccati Differential Equation, Bessel Functions

\section{Introduction}

We consider a special normal form of Riccati equation, $w^{\prime}=z^{n}+w^{2}$, where $w^{\prime}=\mathrm{d} w / \mathrm{d} z$. We prove, for any real number $n$, the equation has a closed analytic solution. We show, the general solution for $n \neq-2$ is a product of $\sqrt{\mathrm{z}^{n}}$ and a combination of Bessel functions of various $n$-dependent orders and arguments. We discuss the variable transformations leading to this general result. Introducing another set of variable transformations conducive to a closed analytic solution, we by-pass the singular behavior of the Bessel functions for $n=-2$. We show, the general solution in the limit of $n \rightarrow-2$ is identical to the solution of $n=-2$.
\end{abstract}

We present the general solution to the Riccati differential equation, $\frac{\mathrm{d} w}{\mathrm{~d} z}=z^{n}+w^{2}$, for arbitrary real number

\section{Procedure}

Riccati equation is given by,

$$
u^{\prime}=a(z)+b(z) u+c(z) u^{2},
$$

where $a(z), b(z)$ and $c(z)$ are analytic functions of $z$. It is known, for $c(z) \neq 0$,

$$
u=\frac{1}{c(z)} w-\frac{b(z)}{2 c(z)}-\frac{c^{\prime}(z)}{2 c^{2}(z)}
$$

transforms (1) into the normal form

$$
w^{\prime}=A(z)+w^{2},
$$

where

$$
A(z)=a c-\frac{b^{2}}{4}+\frac{b^{\prime}}{2}-\frac{3}{4}\left(\frac{c^{\prime}}{c}\right)^{2}-\frac{b}{2} \frac{c^{\prime}}{c}+\frac{1}{2} \frac{c^{\prime \prime}}{c} .
$$

It is the objective of this paper to solve (2) for a special case where $A(z)=z^{n}$. We prove the solution for any real value of $n$ is analytic.

We begin with Euler variable transformation, [1, p. 112], namely $-w(z)=d / d z \ln y(z)$. This linearizes (2)

$$
y^{\prime \prime}+z^{n} y=0,
$$

Multiplying both sides of (3) by $z^{2}$ we compare the result, $z^{2} y^{\prime \prime}+z^{n+2} y=0$ vs.

$z^{2} y^{\prime \prime}+\left(\alpha^{2} \beta^{2} z^{2 \beta}+1 / 4-v^{2} \beta^{2}\right) y=0$ of $[\mathbf{1}$, p. 206] and deduce the following identities

$$
\begin{aligned}
& \beta=(n+2) / 2, v=1 /(2 \beta)=1 /(n+2), \\
& \text { and } \alpha=1 / \beta=2 /(n+2)
\end{aligned}
$$

Furthermore, according to the last reference, the solution of the given equation is, $y=\sqrt{z} f\left(\alpha z^{\beta}\right)$ where $f(\xi)$ is a solution of the Bessel equation of order $v$. Therefore, we conclude the solution to, $z^{2} y^{\prime \prime}+z^{n+2} y=0$ is

$$
y=\sqrt{z}\left[c_{1} J_{v}(\xi)+c_{2} Y_{v}(\xi)\right],
$$

where $J_{v}$ and $Y_{v}$ are the Bessel functions of the first and the second kind of order $v$, with $c_{1}$ and $c_{2}$ being two arbitrary constants. Substituting (4) in Euler transformation and applying the chain differentiation, $\mathrm{d} / \mathrm{d} z=\mathrm{z}^{\beta-1} \mathrm{~d} / \mathrm{d} \xi$. we deduce

$$
-w=\frac{c_{1} J_{v}(\xi)+c_{2} Y_{v}(\xi)+2 z^{\beta}\left[c_{1} J_{v}^{\prime}(\xi)+c_{2} Y_{v}^{\prime}(\xi)\right]}{2 \sqrt{z}\left[c_{1} J_{v}(\xi)+c_{2} Y_{v}(\xi)\right]},
$$

with the prime notation indicating the derivative of the Bessel functions with respect to variable $\xi$. Further- 
more, applying two sets of recurrence relationships $[2, \mathrm{p}$. 361],

$$
\begin{aligned}
& \left.\frac{1}{2}\left[\begin{array}{c}
J(\xi) \\
Y(\xi)
\end{array}\right\}_{v-1}-\left\{\begin{array}{l}
J(\xi) \\
Y(\xi)
\end{array}\right\}_{v+1}\right] \\
& =\left\{\begin{array}{l}
J^{\prime}(\xi) \\
Y^{\prime}(\xi)
\end{array}\right\}_{v}, \frac{\xi}{2 v}\left[\left\{\begin{array}{c}
J(\xi) \\
Y(\xi)
\end{array}\right\}_{v-1}+\left\{\begin{array}{c}
J(\xi) \\
Y(\xi)
\end{array}\right\}_{v+1}\right]=\left\{\begin{array}{c}
J(\xi) \\
Y(\xi)
\end{array}\right\}_{v},
\end{aligned}
$$

simplifies (5)

$$
w=-\sqrt{z^{n}} \frac{c_{1} J_{-\frac{n+1}{n+2}}\left(\frac{2}{n+2} z^{\frac{n+2}{2}}\right)+c_{2} Y_{-\frac{n+1}{n+2}}\left(\frac{2}{n+2} z^{\frac{n+2}{2}}\right)}{c_{1} J_{\frac{1}{n+2}}\left(\frac{2}{n+2} z^{\frac{n+2}{2}}\right)+c_{2} Y_{\frac{1}{n+2}}\left(\frac{2}{n+2} z^{\frac{n+2}{2}}\right)},(6)
$$

And, (6) simplifies to its final form

$$
w=-\sqrt{z^{n}} \frac{C J_{-\frac{n+1}{n+2}}\left(\frac{2}{n+2} z^{\frac{n+2}{2}}\right)+Y_{-\frac{n+1}{n+2}}\left(\frac{2}{n+2} z^{\frac{n+2}{2}}\right)}{C J_{\frac{1}{n+2}}\left(\frac{2}{n+2} z^{\frac{n+2}{2}}\right)+Y_{\frac{1}{n+2}}\left(\frac{2}{n+2} z^{\frac{n+2}{2}}\right)},
$$

With $C=c_{1} / c_{2}$.

We observe that solution (2) is a one-parameter family function.

The $n=-2$ is the pole of the order and the argument of the Bessel functions and needs a special consideration. For $n=-2$ Equation (2) takes the form

$$
w^{\prime}=\frac{1}{z^{2}}+w^{2}
$$

By inspection, $w_{1}=\lambda / z^{2}$ solves (8). Substituting $w_{1}$ in (8) gives $\lambda^{2}+\lambda+1=0$. We select $\lambda=1 / 2(-1+\sqrt{3} i)$ to further the analysis. The standard variable transformation $[1$, p. 50]

$$
w=w_{1}+\frac{1}{v(z)},
$$

gives the general solution. Substituting (9) in (8) yields,

$$
v^{\prime}+2 \frac{\lambda_{1}}{Z} v+1=0,
$$

The solution of (10) is, $v=c / z^{2 \lambda_{1}}-z /\left(2 \lambda_{1}+1\right)$, with $C$ being a constant. The solution to (8) yields,

$$
w=\frac{1}{z}\left[\frac{1}{2}(-1+\sqrt{3} i)+\frac{1}{C z^{-\sqrt{3 i}}-\frac{1}{\sqrt{3} i}}\right],
$$

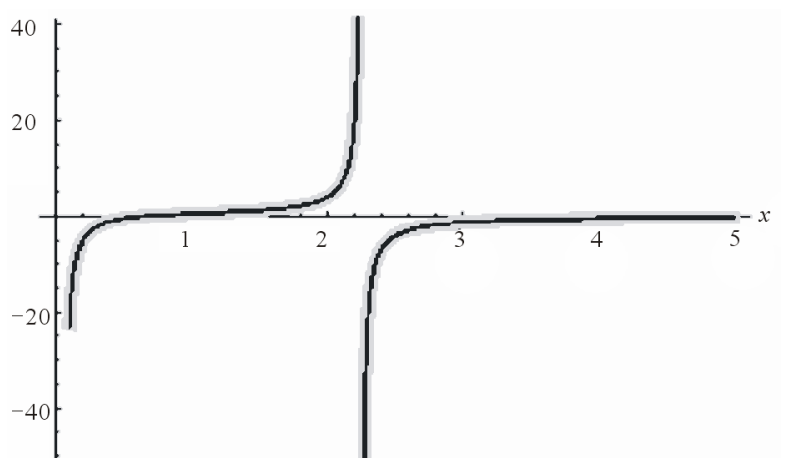

Figure 1. The solid curve is the graph of the solution given in (12) for $c_{1}=1$. The gray curve is the graph of the solution given in (7) for $n=-1.99$ and $C=0.85$.

Applying the identities, $z=e^{\ln z}$ and $\tan z=\frac{1}{i} \frac{e^{i z}-e^{-i z}}{e^{i z}+e^{-i z}}$ and suitably selecting the value of $C=-\frac{i}{\sqrt{3}} e^{-\sqrt{3} c_{1} i}$, becomes

$$
w=\frac{1}{2 z}\left\{-1-\sqrt{3} \cot \left[\frac{\sqrt{3}}{2}\left(\ln z+c_{1}\right)\right]\right\},
$$

We show graphically, see Figure 1, that (7) in the limit of $n \rightarrow-2$ is identical to (12).

\section{A comment and an Acknowledgement}

By applying multiple step variable transformations (not reported in this paper) the author devised a method of solving (2) resulting in (7). The author appreciates the in depth discussion with Prof. Rostamian.

\section{References}

[1] W. E. Boyce and R. C. DiPrima, "Elementary Differential Equations and Boundary Value Problems," 6th Edition, Wiley, New York, 1997.

[2] M. Abramowitz and I. A. Stegun, "Handbook of Mathematical Functions," Dover Publications Inc., New York, 1970. 